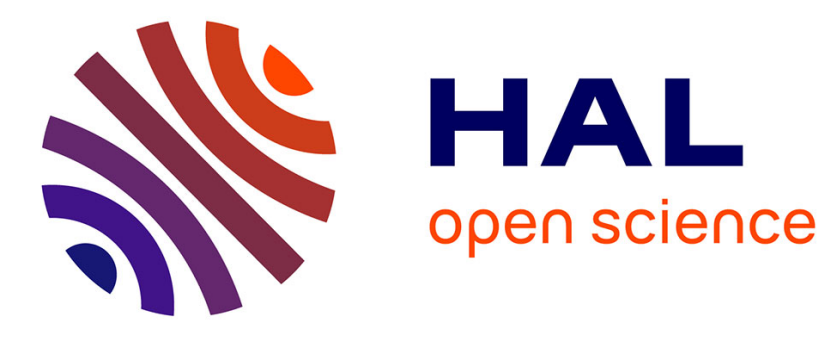

\title{
Die (Drôme) : de la ville ouverte à la ville fortifiée Jacques Planchon
}

\section{To cite this version:}

Jacques Planchon. Die (Drôme): de la ville ouverte à la ville fortifiée. Gallia - Archéologie de la France antique, 2006, Antiquité tardive, haut Moyen Âge et premiers temps chrétiens en Gaule méridionale (première partie): réseau des cités, monde urbain et monde des morts, 63, pp.75-79. 10.3406/galia.2006.3285 . hal-01914296

\section{HAL Id: hal-01914296 \\ https://hal.science/hal-01914296}

Submitted on 8 Jan 2020

HAL is a multi-disciplinary open access archive for the deposit and dissemination of scientific research documents, whether they are published or not. The documents may come from teaching and research institutions in France or abroad, or from public or private research centers.
L'archive ouverte pluridisciplinaire HAL, est destinée au dépôt et à la diffusion de documents scientifiques de niveau recherche, publiés ou non, émanant des établissements d'enseignement et de recherche français ou étrangers, des laboratoires publics ou privés.

\section{(이) $\$$}

Distributed under a Creative Commons Attribution - NonCommercial - NoDerivatives| 4.0 


\title{
DIE (DRÔME)
}

\section{De la ville ouverte à la ville fortifiée}

\author{
Jacques PLANCHON
}

\begin{abstract}
Mots-clés. Rempart, fortification, topographie urbaine, voirie, capitale, colonie.
Résumé. Les vestiges de la ville antique de Die - notamment son rempart - laissent entrevoir l'évolution complexe de cette agglomération gallo-romaine. Vraisemblablement simple relais de bord de voie au début du $I^{e r}$ s., Die acquiert un caractère véritablement urbain avant le début du II ${ }^{e}$ s., et c'est dans une ville ouverte que sont transférées les institutions de la capitale nord des Voconces vers le milieu du II ${ }^{e}$ s. Au III ${ }^{e}$ s., la cité devient colonie et une part importante de l'agglomération s'entoure d'un rempart, laissant de côté certains quartiers périphériques qui semblent être déjà abandonnés.
\end{abstract}

Key-words. Rampart, fortification, topography, road system, chief town, colonia.

Abstract. The complex evolution of the Gallo-Roman town of Die can be observed through the remains of the ancient city - notably its rampart. Small road-relay at the beginning of the $1^{r s t} c$. presumably, Die really obtains its urban features before the early $2^{\text {nd }} c$. and it's in an open town that the institutions of the northern chief town of the Vocontii are being transfered around the middle $2^{\text {nd }} c$. By the $3^{\text {rd }} c$., the city becomes a colony and a large part of the town is surrounded with a wall, leaving aside some outlying quarters, which already seem to be abandoned.

Translation: Isabelle FAUDUET

Schlagwörter. Stadtmauer, Befestigung, Stadttopographie, Straßenbau, Hauptstadt, colonia.

Zusammenfassung. An den Überresten des antiken Die ist die komplexe Entwicklung dieser gallorömischen Ansiedlung ablesbar. Das trifft besonders für die Stadtmauer zu. Die, zu Beginn des ersten Jahrhunderts wahrscheinlich nur eine einfache Straßenstation, besaß bereits vor der Wende zum zweiten Jahrhundert regelrecht städtischen Charakter. Gegen Mitte des zweiten Jahrhunderts erhielt die noch unbefestigte Stadt die zentralen Einrichtungen des nördlichen Hauptortes der Voconces. Im dritten Jahrhundert wird sie Kolonie und eine Stadtbefestigung umschließt einen Großteil der Siedlung. Einige Viertel am ehemaligen Stadtrand, die zu dieser Zeit anscheinend bereits verlassen waren, blieben bei deren Errichtung unberücksichtigt.

Übersetzung: Stefan WIRTH

\section{LA VILLE OUVERTE DU HAUT-EMPIRE}

La ville de Die est située à mi-chemin entre la vallée du Rhône et celle de la Durance, dans les Préalpes drômoises, au sud du massif du Vercors. Sa situation sur la voie médiane reliant le Rhône à l'Italie par les Alpes permet de la considérer, au début du $\mathrm{I}^{\mathrm{er}}$ s. apr. J.-C., comme une simple agglomération secondaire servant d'étape. Les quelques vestiges observés dans le centre-ville et les nécropoles qui l'entourent montrent une évolution de cette station-relais initiale vers un statut plus directement urbain dans le courant du
$\mathrm{I}^{\mathrm{er}}$ s. Son nom, Dea Augusta Vocontiorum, apparaît vers la fin de ce siècle dans l'épigraphie (CIL, XII, 1529 et 1581) ; il est traditionnellement mis en relation avec le nom d'une déesse topique considérée comme éponyme et présente dans un rayon de $20 \mathrm{~km}$ autour du site, Dea Augusta Andarta (CIL, XII, 1554-1560).

$\mathrm{Au}$ début $\mathrm{du} \mathrm{II}^{\mathrm{e}}$ s., l'agglomération semble avoir atteint une superficie de l'ordre de 35 ha, étagée sur quatre niveaux de terrasses surplombant le lit majeur de la Drôme (fig. 37). Ces terrasses sont aménagées et aplanies, comme le montrent les importants murs de soutènement repérés en 


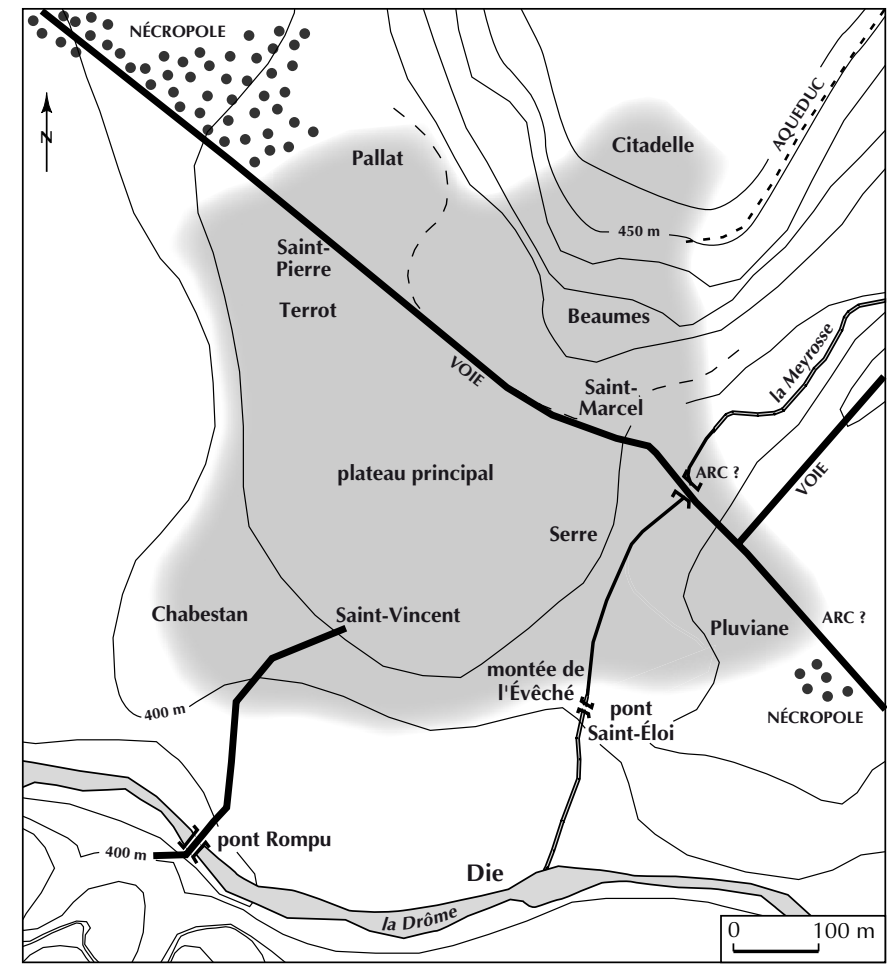

Fig. 37 - Die (Drôme). Extension maximale de l'agglomération au Haut-Empire (DAO J. Planchon, musée de Die et du Diois).

contrebas du plateau de Beaumes. D’imposants blocs architecturaux signalés et observés sur ce plateau indiquent au moins une construction monumentale dont l'identification reste hypothétique, tout comme celle des autres éléments de parure monumentale, par exemple les colonnes de granite gris réutilisées dans le clocher-porche de la cathédrale du $\mathrm{XI}^{\mathrm{e}}$ s. La voie principale est dallée et, bien que marquant une courbe pour respecter la topographie, est assimilée au decumanus major.

La ville de Die acquiert un statut de capitale dans la première moitié du $\mathrm{II}^{\mathrm{e}}$ s., en remplacement de Lucus Augusti/ Luc-en-Diois qui partageait initialement ce statut avec l'autre capitale de la cité fédérée des Voconces, Vasio/Vaison-laRomaine (Pline, HN, III, 37). Bien que mal connue du fait de l'enfouissement des vestiges dû à un important colluvionnement et faute de fouilles, l'agglomération dioise semble présenter tous les éléments de parure des capitales provinciales: thermes monumentaux de Pluviane en périphérie orientale, alimentés par l'un des deux aqueducs de la ville; mosaïques de domus observées pour la plupart dans le centre de l'agglomération, qui abrite également des indices de thermes publics ou privés; voies dallées et places monumentales dont celle repérée à l'ouest de la ville, dans le quartier du Pallat - qui est abandonnée et dont les matériaux sont récupérés vers la fin du $\mathrm{II}^{\mathrm{e}}$ s. (Planchon, 1998, p. 23).

Les raisons du transfert de chef-lieu de Lucus vers Dea sont certainement multiples (Planchon, 2004b), mais rien ne permet de les mettre en relation avec l'accession de Die au statut de colonie, qui intervient probablement plus tard, dans le courant du III $^{\mathrm{e}} \mathrm{s}$. d'après l'inscription Colonia Dea Augusta Vocontiorum sur un sarcophage de cette époque (CIL, XII, 690). En revanche, ce nouveau statut, obtenu à titre honorifique s'il est postérieur à 212 (édit de Caracalla sur la citoyenneté), explique certainement l'édification d'un arc monumental au III ${ }^{\mathrm{e}}$ s. (Küpper-Böhm, 1996, p. 142), dont l'emplacement originel est à restituer à l'entrée orientale de la ville, sur la route de l'Italie : peut-être sur le pont franchissant le ravin de la Meyrosse?

La construction du rempart de Die est traditionnellement attribuée à la charnière des $\mathrm{III}^{\mathrm{e}}-\mathrm{IV}^{\mathrm{e}} \mathrm{s}$., dans le contexte général qui suit les troubles de la fin du $\operatorname{III}^{\mathrm{e}} \mathrm{s}$. : péjoration climatique, raids germaniques et bagaudes, instabilité politique et monétaire. S'il n'est pas possible, faute de documentation, d'établir de lien étroit entre l'édification de l'enceinte urbaine et le statut de colonie attribué à la ville, ces deux faits ne sont certainement pas sans rapport - au moins de façon indirecte.

\section{LA CONSTRUCTION DE L'ENCEINTE AU BAS-EMPIRE}

Le rempart de Die délimite un espace fortifié de 25 ha, pour un périmètre de l'ordre de $1950 \mathrm{~m}$ (fig. 38). Il englobe la partie centrale de l'agglomération préexistante et trois des quatre terrasses initiales, excluant la plus basse. Le tracé irrégulier s'apparente à la forme d'une poire, dont le sommet englobe une portion du plateau dominant la ville, d'où deux segments grossièrement rectilignes descendent en s'élargissant pour ceindre la ville basse en respectant les bords de la terrasse la plus étendue, sur laquelle s'étend la majeure partie de l'agglomération, de part et d'autre de la voie principale.

Les courtines, larges de 3,60 m à 3,90 m, ont une hauteur qui varie de $8 \mathrm{~m}$ à $11 \mathrm{~m}$ en fonction de la topographie, notamment dans les pentes entre le point culminant de l'espace fortifié $(470 \mathrm{~m})$ et son point le plus bas $(400 \mathrm{~m})$. Elles sont constituées de lits successifs de maçonnerie enserrés entre deux parements de petit appareil en calcaire dur régulièrement assisé, où apparaissent par endroits des cordons de briques ou de dalles peu épaisses. Une portion de parement, conservé sur une tour, présente également un 


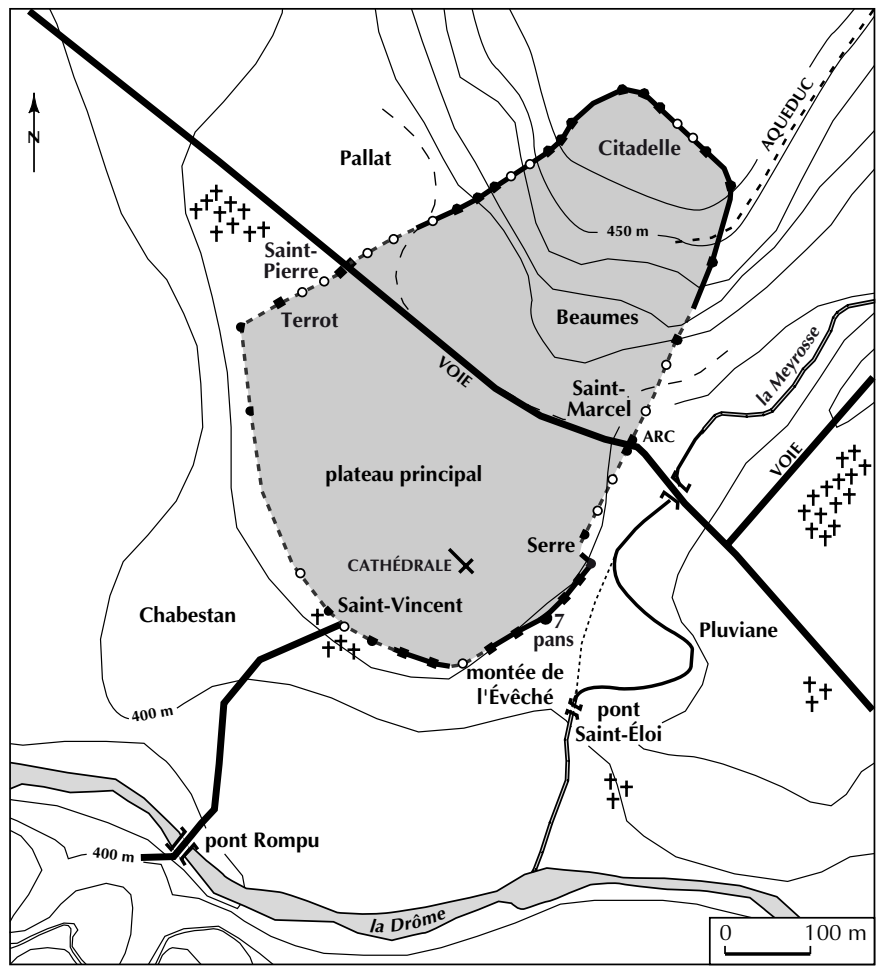

- restitution de tour

tracé antique de la Meyrosse restitué

Fig. 38 - Die (Drôme). Ville fortifiée au Bas-Empire (DAOJ. Planchon, musée de Die et du Diois).

effet de polychromie de briques et de moellons de calcaire alternés en damier sur deux à trois assises. S'il est vrai que ces effets décoratifs sont peu présents sur l'enceinte, il faut cependant considérer que peu de surfaces parementées sont parvenues jusqu'à nous au travers des incessantes destructions et réparations du Moyen Âge, des guerres de religion et $\mathrm{du} \operatorname{XIX}^{\mathrm{e}} \mathrm{s}$; ; en conséquence, il est difficile de généraliser ces observations à l'ensemble du rempart.

Les fondations sont installées en tranchée aveugle ou s'appuient sur les marnes lorsque ces dernières affleurent sous le cailloutis des terrasses. Des blocs de remplois s'y trouvent intégrés par endroits, ainsi que dans les premières assises d'élévation, sans que cette présence soit systématique; on les observe plus fréquemment à la base des tours et dans les pentes.

Une cinquantaine de tours sont restituables sur le tracé de la fortification. Ces tours, pleines à la base, sont munies de talons quadrangulaires et la forme de leur avancée varie en fonction de leur implantation : dans les pentes fortes et au sommet du plateau, ces avancées sont de plan trapé-zoïdal, quadrangulaires en rebord de terrasse et semi-circulaires sur le pourtour de la ville basse. Certaines des tours les mieux conservées, dans le secteur nord-ouest, présentent encore les traces de l'accès au chemin de ronde par une porte donnant sur une salle circulaire de $3 \mathrm{~m}$ de diamètre, éclairée par des fenêtres ouvrant sur l'extérieur de la ville. Faute d'éléments de couronnement conservés, la hauteur des tours est à restituer entre $12 \mathrm{~m}$ et $14 \mathrm{~m}$ au minimum. L'une de ces tours est très particulière: son avancée présente sept pans, chaque angle étant marqué par un chaînage alterné de briques et de moellons de tuf.

Une autre particularité de cette enceinte réside dans sa porte orientale (appelée Saint-Marcel en référence à un évêque du $\mathrm{IV}^{\mathrm{e}} \mathrm{s}$.) qui abrite une partie de l'arc monumental du III $^{\mathrm{e}}$ s. Celui-ci, démonté de son emplacement d'origine, a été remonté pierre par pierre au sein de la porte fortifiée, côté ville (Desaye, 1993, p. 393). Côté campagne, la porte est constituée d'une simple baie enserrée entre deux tours à avancée semi-circulaire. Le couronnement de l'ensemble, démoli avant le XIII ${ }^{\mathrm{e}}$ s., nous est inconnu; on peut sans doute imaginer un passage en arcade reliant les courtines, comme sur l'une des portes d'Autun (Planchon, 2004a, p. 22).

Des clichés pris avant la démolition en 1891 de la porte Saint-Pierre, à l'entrée ouest de la ville, montrent clairement des éléments architecturaux antiques dans son élévation, sans qu'il soit possible de la reconstituer - à l'exception de son claveau sommital, côté extérieur, sur lequel est gravée une tête de taureau vu de trois quarts face répondant aux protomés de l'arc de la porte Saint-Marcel. Cette porte ouest était également enserrée entre deux tours et les fondations de l'une d'entre elles, côté nord, ont pu être observées dans une cave : il s'agissait d'une tour de plan quadrangulaire abritant au rez-de-chaussée une salle accessible intra muros par une petite porte. L'autre tour est également représentée selon un plan quadrangulaire sur le cadastre de 1827 .

Quant aux autres portes, démolies au début $\mathrm{du} \mathrm{XX}^{\mathrm{e}} \mathrm{s}$., rien ne permet d'affirmer ni d'infirmer leur appartenance à la période romaine. On notera cependant que la porte SaintVincent, au sud, donne directement sur le pont Rompu dont les culées et la pile centrale sont attribuables à cette époque. Enfin, deux poternes ont été repérées, protégées chacune dans l'angle d'une tour, sur le plateau sommital.

\section{UNE ÉVOLUTION URBAINE : LE REMPART}

Passant de 35 ha à 25 ha, il est indubitable que la surface de l'agglomération s'est vue réduite lors de la construction de l'enceinte. Des quartiers périphériques sont laissés à l'extérieur, et non des moindres : à l'est, 


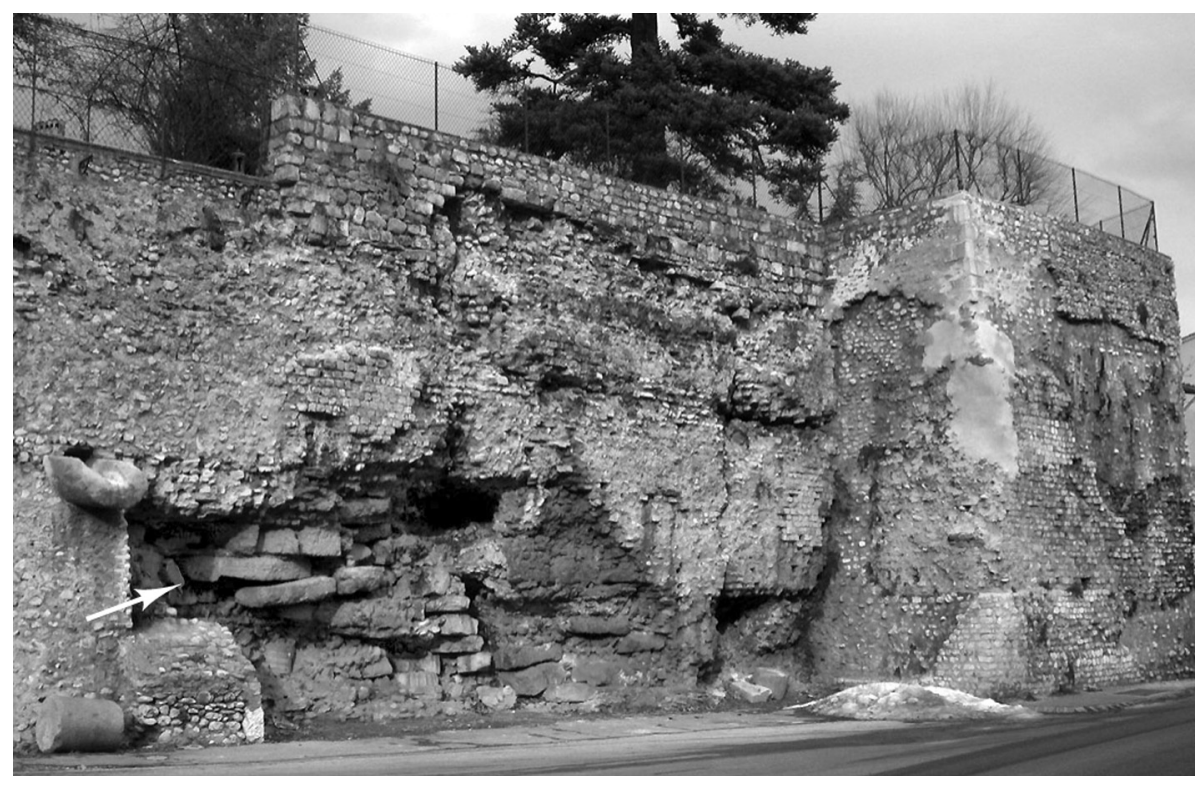

Fig. 39 - Die (Drôme). Dalles de rue en remploi à la base d'une courtine (cliché J. Planchon, musée de Die et du Diois).

les thermes monumentaux de Pluviane, sur un plateau opposé à la ville par rapport au ravin de la Meyrosse; au sud, un quartier d'habitation qui occupait la terrasse inférieure; à l'ouest, l'esplanade monumentale du Pallat, que l'on devine bordée d'édifices de prestige comme le laisse supposer la base de colonne en marbre blanc de 1,60 m de côté qui y a été retrouvée en 1976. En outre, un sondage réalisé en 1997 rue Terrot, à l'est de la ville, a montré que l'enceinte a été implantée à l'emplacement d'un bâtiment préexistant, vraisemblablement une habitation, sans qu'il soit cependant possible de déterminer si celle-ci a été démolie pour l'occasion ou s'il n'en subsistait que des ruines lors de l'édification du rempart (Planchon, 2004c, p. 50). Les observations réalisées au Pallat entretiennent ce doute : l'esplanade monumentale mise au jour lors de la fouille de 1998 est en effet abandonnée dès la fin du $\mathrm{II}^{\mathrm{e}} \mathrm{s}$.; dans le courant du $\mathrm{III}^{\mathrm{e}} \mathrm{s}$., les dalles de cet espace et une partie des élévations sont déjà récupérées et un fossé, creusé durant cette phase, est utilisé comme dépotoir et comblé dès la seconde moitié du III ${ }^{\mathrm{e}}$ s. (Planchon, 1998, p. 20).

Faute de fouilles, il n'est pas possible de dater l'abandon des thermes de Pluviane et du quartier d'habitation de Chabestan. Les deux exemples fouillés à l'est de la ville montrent cependant l'impossibilité de conclure trop hâtivement que ces quartiers périphériques ont été abandonnés du fait de la construction de l'enceinte : l'un au moins d'entre eux était déjà un terrain vague lors de cette construction.
S'il est difficile de préciser les causes de la réduction urbaine, il est en revanche aisé d'en voir les effets sur l'implantation des nécropoles. Les incinérations du HautEmpire dessinent en effet les limites extrêmes de l'extension de l'agglomération le long de la voie. Il est à cet égard intéressant de noter qu'une bonne partie des remplois identifiés dans cette enceinte proviennent de ces nécropoles, qui ont fourni des stèles, simples ou à fronton, des murets d'enclos funéraires, des éléments de mausolées, linteaux inscrits ou blocs architecturaux. Le respect dû aux morts dans la mentalité antique (ou la peur qu'ils inspirent) amène à s'interroger sur la provenance de ces éléments lapidaires: zones de nécropoles peu fréquentées, voire totalement abandonnées? Dans tous les cas, dès le $\mathrm{IV}^{\mathrm{e}} \mathrm{s}$, les inhumations sous tuiles montrent une colonisation par les morts d'espaces qui semblaient jusque-là réservés aux vivants, à Saint-Pierre à l'ouest comme à Saint-Marcel à l'est. Cependant, aucune implantation de tombe n'a été observée jusqu'à présent à proximité immédiate du rempart. Deux hypothèses d'explication sont également plausibles : l'une tient à la topographie, l'enceinte surplombant les pentes limitant la terrasse, l'autre peut être imputée à des réminiscences de poemerium.

Le passage de la ville ouverte à la ville fortifiée influe aussi sur les voies de circulation en créant des passages obligés. La voie principale n'en est pas affectée, mais divers indices laissent supposer une importante transformation des 
autres rues de la ville. Le sondage de la rue Terrot montre qu'une tour de l'enceinte a été construite sur un égout, dont la couverture a été profondément modifiée à cette occasion. L'hypothèse de restitution d'une voie au-dessus de cet égout, bouchée par l'édification du rempart (Planchon, 2004c, p. 52), va dans le même sens qu'une étude antérieure qui confrontait l'implantation des tours au débouché des rues dans ce quartier oriental de la ville (Pietri, 1933, p. 132). Dans deux autres secteurs, au Serre et à la montée de l'Évêché (fig. 39), une accumulation de dalles de rue dans une portion réduite des fondations du rempart semble indiquer que, là encore, des voies ont été fermées par cette construction, les dalles ayant pu être réuti-lisées sur place lors de la mise en ouvre des fondations.

$$
\text { * } *
$$

Le cas de Die cadre assez mal avec la généralisation habituelle du phénomène des enceintes du Bas-Empire enserrant une ville réduite, et édifiées à partir des éléments arrachés aux quartiers périphériques abandonnés. Tout d'abord dans la mesure où la surface fortifiée, 25 ha, reste importante et se démarque nettement d'autres exemples plus caractéristiques de Gaule Lyonnaise ou Belgique. Par ailleurs, l'exemple de l'esplanade monumentale du Pallat, devenue terrain vague avant la construction du rempart, repose la question de ces fameux quartiers périphériques : le tracé de l'enceinte ne vient ici qu'entériner une situation de fait. Dans cette optique, les remplois présents dans cette enceinte pourraient également être considérés comme les témoins d'une crise urbaine, plutôt qu'interprétés comme les vestiges d'édifices volontairement sacrifiés.

Il semble préférable, dans ce cas précis, de voir la surface fortifiée comme le résultat d'une régression urbaine, entamée vers la fin $d u \mathrm{II}^{\mathrm{e}} \mathrm{s}$. et profondément inscrite dans le paysage urbain dès le $\mathrm{III}^{\mathrm{e}} \mathrm{s}$. Il est cependant étonnant de constater que cette régression est concomitante au transfert de la capitale : c'est donc une ville déjà marquée par cette crise qui accède au statut de colonie et se pare d'un arc monumental, puis d'une puissante fortification : intéressant paradoxe. 